\title{
Die Spiroergometrie in der arbeitsmedizinischen Eignungsuntersuchung und Begutachtung
}

\section{Cardiopulmonary Exercise Testing in Occupational Medical Fitness Examination and Assessment}

Autoren

Institute

\author{
A. M. Preisser ${ }^{1}$, U. Ochmann ${ }^{2}$
}

${ }^{1}$ Universitätsklinikum Hamburg-Eppendorf, Zentralinstitut für Arbeitsmedizin und Maritime Medizin

(Direktor Prof. Dr. X. Baur), Klinische Arbeitsmedizin

${ }^{2}$ Institut und Poliklinik für Arbeits,- Sozial- und Umweltmedizin (Direktor Prof. Dr. D. Nowak),

Klinikum der Universität München LMU eingereicht 5.8.2011 akzeptiert nach Revision 18. 10. 2011

Bibliografie

Dol http://dx.doi.org/

10.1055/s-0031-1291463

Pneumologie 2011; 65: 662-670

(c) Georg Thieme Verlag KG

Stuttgart · New York

ISSN 0934-8387

Korrespondenzadresse Alexandra M. Preisser

Universitätsklinikum HamburgEppendorf, Zentralinstitut für Arbeitsmedizin und Maritime Medizin, Klinische Arbeitsmedizin Seewartenstraße 10 20459 Hamburg

a.preisser@uke.de

\section{Zusammenfassung \\ $\nabla$}

Die arbeitsmedizinische und pneumologische Begutachtung hat zwei wesentliche Ziele: die Erstellung einer Diagnose mit an Sicherheit grenzender Wahrscheinlichkeit und die Klärung eines Kausalzusammenhangs mit arbeitsbedingten aktuellen oder früheren Expositionen zu irritativ-toxischen, allergisierenden oder fibrosierend wirksamen Stäuben, Gasen, Schweißrauchen oder Mineralfasern. Vor allem für Krankheitsbilder, die mit einer Belastungsdyspnoe einhergehen, ist die Diagnostik in Ruhe mittels Spirometrie, Bodyplethysmografie, Blutgasanalyse, EKG und Echokardiografie nur eingeschränkt geeignet. Der Artikel benennt die Indikationen zur Spiroergometrie in der Arbeitsmedizin, erklärt die zugehörigen Messparameter und ihren differenzialdiagnostischen Wert einschließlich der Fluss-Volumen-Kurve unter Belastung und der alveolo-arteriellen Sauerstoffdifferenz. Die diagnostische Qualität der gemessenen Sauerstoffaufnahme bei Dauer- und Spitzenbelastung im Vergleich zur ermittelten Wattleistung auf dem Fahrradergometer wird dargestellt. Charakteristische Spiroergometriebefunde werden im Hinblick auf ihre differenzialdiagnostische Bedeutung erläutert. Weiterhin wird die Bedeutung der Spiroergometrie zur Bemessung der berufskrankheitenbedingten Funktionseinschränkungen (klinischer Anteile der Minderung der Erwerbsfähigkeit) dargestellt.

\section{Einleitung}

Die Spiroergometrie ist eine sensitive Methode zur kardiopulmonalen Leistungsdiagnostik. Sie wird eingesetzt u.a. zur Einschätzung der Operabilität bei pulmonalen, kardialen und anderen Grunderkrankungen und zur Erfassung der maximalen Leistungsfähigkeit, der aerob-anaeroben Schwelle und der Dauerleistungsgrenze bei Sportlern. Dem entsprechend ist sie gleichfalls in der

\section{Abstract \\ $\nabla$}

Medical expert opinion by occupational physicians and pneumologists has two main objectives: making a diagnosis with probability bordering on certainty and clarifying a causal relationship to a present or former occupational exposure to irritant toxic, allergenic or fibrosing dusts, gases, welding fumes or mineral fibres. Especially for conditions that are associated with exertional dyspnea, the diagnosis at rest using spirometry, body plethysmography, pulmonary function test, blood gas analysis, electrocardiogram and echocardiography is of limited use. This paper identifies the indications for cardiopulmonary exercise testing (CPET) in occupational medicine, explains the related measurements and their differential diagnostic value with special consideration of the flow-volume curve under exercise as well as the alveolar-arterial oxygen gradient. Diagnostic statements on the relevance of oxygen uptake measured at continuous and peak load compared to the wattage ascertained on the bicycle ergometer are presented. Characteristic CPET findings are explained in terms of their differential diagnostic significance. Furthermore, the importance of CPET for the assessment of occupational diseaserelated functional loss (clinical proportions in the reduction of working capacity) is shown.

Arbeitsmedizin dafür geeignet, einen differenzierten Einblick in die kardiopulmonale Belastbarkeit eines Arbeitnehmers zu geben. Für die arbeitsmedizinische Begutachtung muss eine Belastungsdyspnoe in der Beweisführung für eine Berufskrankheit differenziert und korrekt eingeordnet werden. Die Diagnostik mittels Spirometrie und Blutgasanalyse, EKG und Echokardiografie kann Funktionslimitierungen des Patienten in Ruhe, meist als Korrelat eines weiter fortgeschrit- 
Tab.1 Berufskrankheiten, in deren Begutachtung die Unfallversicherungsträger die Spiroergometrie zur Diagnostik und MdE-Bemessung empfehlen.

\begin{tabular}{|c|c|}
\hline \multicolumn{2}{|c|}{ Asbestbedingte Berufskrankheiten (nach [4]) } \\
\hline BK-Nummer & Erläuterung \\
\hline 4103 & $\begin{array}{l}\text { Asbeststaublungenerkrankung (Asbestose) oder durch } \\
\text { Asbeststaub verursachte Erkrankung der Pleura }\end{array}$ \\
\hline 4104 & $\begin{array}{l}\text { Lungenkrebs oder Kehlkopfkrebs } \\
\text { - in Verbindung mit Asbeststaublungenerkrankung } \\
\text { (Asbestose) } \\
\text { - in Verbindung mit durch Asbeststaub verursachter } \\
\text { Erkrankung der Pleura oder } \\
\text { - bei Nachweis der Einwirkung einer kumulativen } \\
\text { Asbestfaserstaub-Dosis von mind. } 25 \text { Faserjahren }\end{array}$ \\
\hline 4114 & $\begin{array}{l}\text { Lungenkrebs durch das Zusammenwirken von Asbest- } \\
\text { faserstaub und polyzyklischen aromatischen Kohlen- } \\
\text { wasserstoffen bei Nachweis der Einwirkung einer kumu- } \\
\text { lativen Dosis, die einer Verursachungswahrscheinlichkeit } \\
\text { von mindestens } 50 \text { Prozent entspricht }\end{array}$ \\
\hline & $\begin{array}{l}\text { Lungenkrebserkrankung nach Resektion und „Heilungs- } \\
\text { bewährung“ }\end{array}$ \\
\hline \multicolumn{2}{|c|}{ Silikosen (nach [5]) } \\
\hline 4101 & Quarzstaublungenerkrankung (Silikose) \\
\hline 4102 & $\begin{array}{l}\text { Quarzstaublungenerkrankung in Verbindung mit aktiver } \\
\text { Lungentuberkulose (Siliko-Tuberkulose) }\end{array}$ \\
\hline \multicolumn{2}{|c|}{$\begin{array}{l}\text { Obstruktive Atemwegserkrankungen } \\
\text { (Empfehlung der Spiroergometrie in der Differenzialdiagnostik, } \\
\text { nach Reichenhaller Empfehlung, Verabschiedung Ende 2011) }\end{array}$} \\
\hline 1315 & $\begin{array}{l}\text { Erkrankungen durch Isocyanate, die zur Unterlassung } \\
\text { aller Tätigkeiten gezwungen haben, die für die Entste- } \\
\text { hung, die Verschlimmerung oder das Wiederaufleben } \\
\text { der Krankheit ursächlich waren oder sein können }\end{array}$ \\
\hline 4301 & $\begin{array}{l}\text { Durch allergisierende Stoffe verursachte obstruktive } \\
\text { Atemwegserkrankungen (einschließlich Rhinopathie), } \\
\text { die zur Unterlassung aller Tätigkeiten gezwungen haben, } \\
\text { die für die Entstehung, die Verschlimmerung oder das } \\
\text { Wiederaufleben der Krankheit ursächlich waren oder sein } \\
\text { können }\end{array}$ \\
\hline 4302 & $\begin{array}{l}\text { Durch chemisch-irritativ oder toxisch wirkende Stoffe } \\
\text { verursachte obstruktive Atemwegserkrankungen, die zur } \\
\text { Unterlassung aller Tätigkeiten gezwungen haben, die für } \\
\text { die Entstehung, die Verschlimmerung oder das Wieder- } \\
\text { aufleben der Krankheit ursächlich waren oder sein können }\end{array}$ \\
\hline
\end{tabular}

tenen Krankheitsstadiums, aufzeigen. Sie ermöglicht jedoch keine eindeutige Abgrenzung von einer sich ausschließlich unter körperlicher Belastung darstellenden kardiopulmonalen Limitierung und häufig keine sicheren Rückschlüsse auf das ursächliche Grundleiden der eingeschränkten Belastbarkeit.

In diesem Artikel sollen insbesondere die Bedeutung und die Praxis der Spiroergometrie in der arbeitsmedizinischen Begutachtung erläutert werden; er ist eine Weiterführung unseres früheren Artikels zu „Pneumologischen Belastungsuntersuchungen in der Arbeitsmedizin“ [1].

\section{Indikationen}

Die Spiroergometrie findet Anwendung bei arbeitsmedizinischen Eignungsuntersuchungen besonderer Berufsgruppen (Feuerwehr, Berufstaucher, Druckluftarbeiter), in sozialrechtlichen Verfahren zur individuellen Klärung von Berufs- oder Erwerbsunfähigkeit sowie in der Begutachtung von Berufskrankheiten zur Diagnosesicherung und der Bewertung des Ausmaßes des Funktionsschadens (MdE). Die verschiedenen die Atemwege und das Lungenparenchym betreffenden Berufskrankheiten zeigen Auf-
Tab.2 Berufskrankheiten, in deren Begutachtung die Spiroergometrie zur MdE-Bemessung herangezogen werden kann, analog den Empfehlungen der Unfallversicherungsträger.

\begin{tabular}{|c|c|}
\hline \multicolumn{2}{|c|}{$\begin{array}{l}\text { Berufskrankheiten mit vornehmlich restriktiver Ventilationsstörung } \\
\text { (Auswahl) }\end{array}$} \\
\hline BK-Nummer & Erläuterung \\
\hline 4115 & $\begin{array}{l}\text { Lungenfibrose durch extreme und langjährige Einwirkung } \\
\text { von Schweißrauchen und Schweißgasen - (Siderofibrose) }\end{array}$ \\
\hline 4201 & Exogen-allergische Alveolitis \\
\hline \multicolumn{2}{|c|}{$\begin{array}{l}\text { Berufskrankheiten mit gemischt obstruktiv-restriktiven } \\
\text { Ventilationsstörungen }\end{array}$} \\
\hline 1110 & Erkrankungen durch Beryllium oder seine Verbindungen \\
\hline \multicolumn{2}{|c|}{ Weitere obstruktive Atemwegserkrankungen } \\
\hline 4111 & $\begin{array}{l}\text { Chronische obstruktive Bronchitis oder Emphysem von } \\
\text { Bergleuten unter Tage im Steinkohlenbergbau bei Nach- } \\
\text { weis der Einwirkung einer kumulativen Dosis von in der } \\
\text { Regel } 100 \text { Feinstaubjahren }[(\mathrm{mg} / \mathrm{m} 3) \text { x Jahre] }\end{array}$ \\
\hline \multicolumn{2}{|c|}{$\begin{array}{l}\text { Lungenkrebserkrankungen nach Resektion und } \\
\text { „Heilungsbewährung“ }\end{array}$} \\
\hline 4112 & $\begin{array}{l}\text { Lungenkrebs durch die Einwirkung von kristallinem } \\
\text { Siliziumdioxid }\left(\mathrm{SiO}_{2}\right) \text { bei nachgewiesener Quarzstaub- } \\
\text { lungenerkrankung (Silikose oder Siliko-Tbc) }\end{array}$ \\
\hline 4113 & $\begin{array}{l}\text { Lungenkrebs durch polyzyklische aromatische Kohlen- } \\
\text { wasserstoffe bei Nachweis der Einwirkung einer kumula- } \\
\text { tiven Dosis von mindestens } 100 \text { Benzo[a]pyren-Jahren } \\
{[(\mu \mathrm{g} / \mathrm{m} 3) \times \text { Jahre] }}\end{array}$ \\
\hline 2402 & $\begin{array}{l}\text { Erkrankungen durch ionisierende Strahlen (z. B. Lungen- } \\
\text { krebs durch Radon) }\end{array}$ \\
\hline
\end{tabular}

fälligkeiten mit vornehmlich restriktiven oder obstruktiven Ventilationsmustern, aber auch Mischbilder oder Einschränkungen, bei denen eine Gasaustauschstörung im Vordergrund steht. Die Spiroergometrie wird inzwischen von verschiedenen Fachverbänden in der Begutachtung, insbesondere zur Differenzialdiagnostik, einiger Berufskrankheiten empfohlen (siehe $\bullet$ Tab.1). Es stehen derzeit zwei von der Deutschen Gesellschaft für Arbeitsmedizin und Umweltmedizin e.V. (DGAUM) gemeinsam mit der Deutschen Gesellschaft für Pneumologie und Beatmungsmedizin e.V. (DGP) und weiteren Fachgesellschaften entwickelte Leitlinien zur Verfügung: „Diagnostik und Begutachtung der BK 4101 Quarzstaublungenerkrankung (Silikose)“ [2] und „Diagnostik und Begutachtung asbestbedingter Berufskrankheiten“" [3].

In den von der Deutschen Gesetzlichen Unfallversicherung (DGUV) herausgegebenen Begutachtungs-Empfehlungen, der Falkensteiner Empfehlung (Asbestbedingte Erkrankungen) [4] und der Bochumer Empfehlung (Silikose) [5], sind die medizinischen Inhalte der AWMF-Leitlinien weitgehend übernommen und um juristische Aspekte ergänzt. Das speziell für berufsbedingte obstruktive Atemwegserkrankungen (BK-Nummer 1315, 4301, 4302, 4111) entwickelte Reichenhaller Merkblatt [6] wird derzeit unter Federführung der DGUV für eine aktualisierte Fassung (Reichenhaller Empfehlung) (2011) grundlegend überarbeitet unter Berücksichtigung der hierzu vorliegenden Aktualisierungsvorschläge [7].

\section{Messparameter der Spiroergometrie}

Die Basisparameter in der Spiroergometrie sind das Atemminutenvolumen $\dot{\mathrm{V} E}$ [Liter/min], die Sauerstoffaufnahme $\dot{\mathrm{VO}}_{2}$ [Liter/ min], die Kohlendioxidabgabe $\dot{\mathrm{V}} \mathrm{CO}_{2}$ [Liter/min], die Herzfre- 


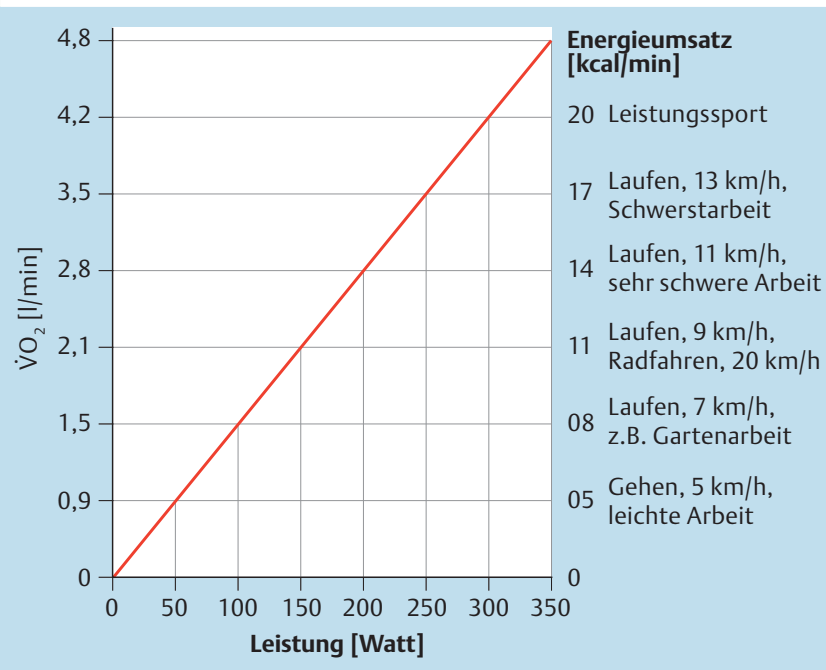

Abb. 1 Lineare Beziehung von $\dot{\mathrm{VO}}_{2}$ zu Leistung in Watt (aus [11]).

quenz [ $1 / \mathrm{min}]$ und die Leistung [Watt] am Fahrradergometer. Diese werden ergänzt durch die Aufzeichnung der Strömungskurve (Fluss-Volumen-Kurve) in Ruhe und unter Belastung (Intrabreath) sowie die Bestimmung der Blutgase und der alveoloarteriellen Sauerstoffdifferenz (PA-a, $\mathrm{O}_{2}$ oder $\mathrm{AaDO}_{2}$ ). In der arbeitsmedizinischen Begutachtung sind Fluss-Volumen-Kurve und $\mathrm{AaDO}_{2}$ wesentlich: Strömungskurve und Blutgase werden zu verschiedenen Zeitpunkten (vorab, während leichter und maximaler sowie nach der Belastung) gemessen. Die $\mathrm{AaDO}_{2}$ wird aus dem Sauerstoff- und Kohlendioxidgehalt in der ausgeatmeten Luft zum jeweiligen Zeitpunkt der Blutentnahme und den dazugehörigen Blutgasen errechnet. Aufgrund der fehlenden direkten Anbindung des Blutgasanalysators an den Computer der Spiroergometrie-Einheit ist derzeit die Berechnung und damit die $\mathrm{Er}$ kennung einer pathologisch erhöhten $\mathrm{AaDO}_{2}$ erst nach Beendigung der Belastung möglich.

Allgemein kann Leistung über die am Fahrradergometer erbrachte Watt-Zahl bestimmt werden. Diese Watt-Zahl stellt jedoch nur die an der Kurbel aufgenommene Arbeit dar. Genauer gelingt die Leistungsbestimmung des gesamten Organismus über den Verbrauch des Sauerstoffs bei der Verbrennung von Kohlenhydraten, Fetten und in geringem Maße Eiweißen. Der für diese Leistung benötigte Verbrauch wird über die Sauerstoffaufnahme gemessen und über die maximal erreichte Sauerstoffaufnahme $\mathrm{VO}_{2 \max }$ beurteilt. Wattleistung und Sauerstoffaufnahme stehen in einer linearen Beziehung $(\bullet$ Abb. 1) [9-12].

Auch andere Belastungsformen wie Gehen, Rudern oder berufliche körperliche Leistungen lassen sich durch die Sauerstoffaufnahme unabhängig von der Belastungsart bestimmen und in den Vergleich zu Sollwerten setzen. Hierzu wurden bisher verschiedene Sollwertempfehlungen publiziert. Häufigste Anwendung finden die Normwerte von Hansen, Sue \& Wasserman [13]; jedoch ist ihr Aussagewert in höheren Altersgruppen und insbesondere bei Frauen unzureichend. Die neu publizierten Normwerte der Greifswalder SHiP (Study of Health in Pomerania) [14] werden in Deutschland zunehmend als Referenz empfohlen; ihre Integration in die Auswertungsprogramme der SpiroergometrieHersteller ist bisher allerdings noch nicht zufriedenstellend gelöst.

\section{Durchführung}

$\nabla$

Bestimmend für die Art der Durchführung der Spiroergometrie sind der jeweilige arbeitsmedizinische Anlass bzw. das Ziel der Untersuchung. Bei Eignungsuntersuchungen entsprechend den berufsgenossenschaftlichen Vorgaben wird die Belastungsleistung sitzend auf dem Fahrradergometer mit einer Leistungssteigerung von 25 Watt je 2 Minuten ermittelt [15].

Die Untersuchung zur arbeitsmedizinischen Begutachtung wird nach einem Rampenprotokoll durchgeführt. Sie kann auch, je nach Gesundheitszustand des Untersuchten, in halbliegender Position erfolgen. Im Rampenprotokoll wird die Belastung nahezu kontinuierlich gesteigert. In der arbeitsmedizinischen Begutachtung sollte zumindest eine submaximale Leistung angestrebt werden (entsprechend ca. $80 \%$ des Sollwertes von $\dot{\mathrm{V}}_{2 \max }$ ), für Eignungsuntersuchungen ist die Ermittlung der maximalen Leistungsfähigkeit erforderlich. Die Gesamtbelastungsdauer soll in beiden Fällen bei ca. 8 bis maximal 12 Minuten liegen. In der Stufenbelastung wird die Anfangsleistung, bei der Rampenbelastung die Steigerung der Wattleistung pro Minute in Abhängigkeit von der erwarteten Maximalleistung gewählt. Eine geschlechterdifferenzierte Übersicht zur maximal erschöpfenden körperlichen Leistung (Watt $=\mathrm{kg}$ (Körpergewicht) $\times 3$ für Männer $/ \times 2,5$ für Frauen, jeweils minus $10 \%$ je Altersdekade ab 30 . LJ) gibt Reiterer 1975, nachgedruckt in BG-Grundsätze [15].

Ob die dem Probanden maximal mögliche Sauerstoffaufnahme $\left(\mathrm{V}_{2 \max }\right)$ erreicht wurde, zeigt sich durch eine Plateaubildung der $\mathrm{VO}_{2}$-Kurve am Ende der Belastungssteigerung. Man kann ohne Wertung der Ausbelastung die erreichte maximale Sauerstoffaufnahme auch mit $\mathrm{VO}_{2 \text { peak }}$ bezeichnen ${ }^{1}$. Ein entscheidender Vorteil der Spiroergometrie ist, dass durch die Ermittlung der $\dot{\mathrm{VO}}_{2}$ an der aerob-anaeroben Schwelle $\left(\dot{\mathrm{VO}}_{2, \mathrm{AT}}\right)$, die Leistungsfähigkeit auch ohne Maximalbelastung beurteilt werden kann.

\section{Pneumologische Bewertung in arbeitsmedizinischen Eignungsuntersuchungen} $\nabla$

Bei Eignungsuntersuchungen nach ArbmedVV für schweren Atemschutz [G 26.3], nach Druckluftverordnung für Berufstaucher und Arbeit im Überdruck [G 31] ist die Spiroergometrie sinnvoll zur Beurteilung der maximalen Leistungsfähigkeit.

Nach den Berufsgenossenschaftlichen Grundsätzen ist derzeit nur eine Ergometrie vorgesehen, bei der die Leistungsfähigkeit als PWC (Physical work capacity) bestimmt wird; abgeleitet aus der Wattleistung pro kg Körpergewicht (KG) bei einer Herzfrequenz von 150 oder $170 / \mathrm{min}$. Unberücksichtigt bleibt hierbei jedoch das zusätzliche Gewicht, welches ein Feuerwehrmann mit Schutzkleidung und Atemgerät zu tragen hat, bzw. der zusätzliche Atemwegswiderstand, der durch schweren Atemschutz oder Tauchausrüstung zu überwinden ist. Durch die spiroergometrische Erfassung der Sauerstoffaufnahme kann die Leistung des Organismus insgesamt, also das Zusammenwirken von Muskulatur, Herz-Kreislaufsystem und Lunge beurteilt werden. Pneumologische Erkrankungen (ein belastungsinduziertes Asthma oder eine bisher unerkannte Gasaustauschstörung), die hier eine Eignung ausschließen, können festgestellt werden.

\footnotetext{
${ }^{1}$ Die Begriffe $\dot{\mathrm{V}}_{2 \text { peak }}$ und $\dot{\mathrm{VO}}_{2 \text { max }}$ werden in dieser Betrachtung gleichgesetzt und bedeuten „maximal erreichte O2-Aufnahme“. Für Details siehe [11].
} 
In der Auswertung der Spiroergometrie wird die maximale Sauerstoffaufnahme als Parameter für die Beurteilung der kardiopulmonalen Leistungsfähigkeit bewertet. Abweichungen von mehr als $20 \%$ unter Sollwert werden als pathologisch eingestuft. $\mathrm{Zu}-$ sätzlich kann auch hier, wie in der Ergometrie, die maximale Pulsfrequenz ermittelt und in Beziehung zu Körpergewicht und Wattleistung gesetzt und ergänzend die PWC bestimmt werden. Untersuchungen zur realen Sauerstoffaufnahme mit schwerem Atemschutz werden derzeit auf einer Feuerwehr-Übungsstrecke von Heblich und Mitarbeitern in Kiel durchgeführt [16].

\section{Beurteilung der Spiroergometrie in der arbeitsmedizi- nischen Begutachtung von Berufskrankheiten (BK) $\nabla$}

Die Spiroergometrie wird in der arbeitsmedizinischen Begutachtung zur Verifizierung einer Atemwegs- oder Lungenerkrankung, vor allem bei Grenzfällen eingesetzt, wenn in den Ruhemessungen noch keine oder nur geringgradige pathologische Veränderungen nachweisbar sind. Sie dient hier der Differenzierung zwischen pulmonalen Funktionseinschränkungen und anderen leistungsmindernden Erkrankungen des Herzens, des neuromuskulären oder des Kreislauf-Systems. Dies ist bedeutsam für den Nachweis der pulmonalen Erkrankung („Vollbeweis“ der Erkrankung) und deren funktionelle Auswirkungen. Die Spiroergometrie gibt außerdem gute Hinweise auf weitere limitierende Faktoren wie die Mitarbeit.

Die im Berufskrankheiten-Verfahren festzusetzende Minderung der Erwerbsfähigkeit (MdE) bezieht sich auf die durch den Funktionsschaden bedingte verminderte Möglichkeit des Patienten, am allgemeinen Erwerbsleben teilzunehmen. In der Bewertung des medizinischen Anteils der MdE unterstützt die Spiroergometrie die Beurteilung des Umfangs der BK-bedingten kardiopulmonalen Funktionseinschränkung und dient der Abgrenzung BK-bedingter von BK-unabhängigen funktionellen Einschränkungen. Kompensierende (z.B. Motivation, arteriovenöse Ausschöpfung, erhöhtes Hämoglobin) und dekompensierende Faktoren (z. B. dynamische Lungenblähung, Diffusionsstörung) können die gemessene Leistungsfähigkeit beeinflussen. Die Beurteilung von Ruhedaten alleine berücksichtigt diese Aspekte nicht.

Kardiale Erkrankungen als Ursache einer Leistungseinschränkung und Belastungsdyspnoe gehen gleichfalls mit erniedrigter maximaler Sauerstoffaufnahme einher und können mit Hilfe des EKG, der Herzfrequenz, der systolischen Blutdruckwerte und der Sauerstoffsättigung im Verlauf der Belastung abgegrenzt werden. Weitere Hinweise sind Erniedrigungen mit frühzeitigen Plateaubildungen von der Sauerstoffaufnahme pro Herzschlag (Sauerstoffpuls) und der Sauerstoffaufnahme pro geleistete Watt-Einheit.

Die Bestimmung der aerob-anaeroben Schwelle und der Zeit, die danach unter anaeroben Bedingungen weiter belastet werden kann, hilft bei der Beurteilung von Trainingszustand, Patientenmotivation und Anstrengungsbereitschaft, soweit keine anderen pulmonalen oder kardialen Gründe für den verfrühten Abbruch der Belastung sprechen. Grundsätzlich kann bei fortgeschrittenen Erkrankungen eine pulmonale oder kardiale Limitierung zu einem Abbruch der Untersuchung führen, bevor ein Übergang der aeroben zur anaeroben Energiebereitstellung erkennbar ist. Über den mitarbeitsunabhängigen Parameter der Sauerstoffaufnahme an der aerob-anaeroben Schwelle $\left(\dot{\mathrm{VO}}_{2, \mathrm{AT}}\right)$ im Verhältnis zum maximalen Sollwert kann die Leistungsfähigkeit auch ohne
Erreichen der individuell maximalen Sauerstoffaufnahme beurteilt werden.

In Einzelfällen kann die Spiroergometrie auch zur Einschätzung des Leistungsvermögens von Patienten mit berufsbedingten Krebserkrankungen nach Krankheitsstabilisierung sowohl bei Tumoren der Lunge als auch bei weiteren, das Herz-Kreislaufsystem potenziell beeinträchtigenden Tumorerkrankungen, wie z.B. Krebserkrankungen des blutbildenden Systems durch Benzol, beitragen. Auch bei der Abklärung von funktionellen Auswirkungen einer Trachealstenose, sei es nach Arbeitsunfall oder als Intubationsfolge durch Tracheomalazie oder durch Rekurrensparese, liefert die Spiroergometrie entscheidende Informationen.

\section{Bewertung der spiroergometrischen Daten in der} Begutachtung bronchopulmonaler Berufskrankheiten Die Belastbarkeit beim Gesunden wird limitiert durch die Schlagleistung des Herzens und den Trainingsstand der Muskulatur. Die Atemvolumina sind dagegen nahezu nicht trainierbar. Pulmonale Reserven sind beim Lungengesunden und Normaltrainierten in der Regel auch unter maximaler Belastung noch vorhanden, bei pathologischer Funktionseinschränkung sind sie unter Belastung aufgebraucht.

Berufsbedingte bronchopulmonale Erkrankungen lassen sich in Erkrankungen mit vornehmlich obstruktiven oder restriktiven Ventilationsstörungen differenzieren ( $\bullet$ Tab.2); Mischbilder (v.a. bei der Silikose anzutreffen) sind möglich (siehe Beispiel). Mittels Spiroergometrie sind Obstruktion und Restriktion als Grund der Leistungslimitierung anhand der Parameter von einer kardial bedingten Limitierung und/oder einem Trainingsmangel abgrenzbar.

Bei den obstruktiven Atemwegserkrankungen liegt in Ruhe nicht selten eine Hypoxämie vor, bedingt durch eine Perfusions-Ventilations-Verteilungsstörung. Bereits unter leichter Belastung kommt es zu einem Ausgleich der Blutgase und der $\mathrm{AaDO}_{2}$; die Bestimmung dieser Parameter sollte daher unter noch geringer Belastung, in der Praxis meist ca. 3-4 Minuten nach Beginn des Belastungstestes, erfolgen. Unter maximaler Belastung können wieder ein Abfall des Sauerstoffpartialdrucks im Blut und/oder eine Erhöhung der $\mathrm{AaDO}_{2}$ auftreten, die auf ein Emphysem mit Gasaustauschstörung hinweisen.

Atemwegsobstruktionen zeigen sich durch eine Strömungslimitierung des exspiratorischen Flusses (z.B. BK 4301). Hierzu wird die vorher in Ruhe reproduzierbar aufgezeichnete Fluss-Volumen-Kurve (entsprechend ATS/ERS-Kriterien) mit den unter Belastung registrierten Atemkurven (Intrabreath-Kurven) verglichen. Eine schwere Obstruktion führt unter Belastung zur Vergrößerung des endexspiratorischen Lungenvolumens, erkennbar in der Verschiebung der Fluss-Volumen-Kurve mit Vergrößerung des end-exspiratorischen Volumens (EELV). Dies wird als „dynamische Blähung“ bezeichnet (siehe Abb.2). Die inspiratorische Kapazität wird hierbei zunehmend aufgebraucht. Zur Beurteilbarkeit der Intrabreath-Kurven unter Belastung im Vergleich mit den vorher in Ruhe aufgezeichneten Spirometrie-Kurven und -Werten ist wesentlich, dass letztere das bestmögliche reproduzierbare Ergebnis (nach ATS/ERS-Qualitäts-Kriterien unter optimaler Patientenmotivation) darstellen. Kann dies nicht gewährleistet werden, können Einschränkungen vorgetäuscht sein: zum einen durch eine Vergrößerung des EELV und hierdurch vorzeitigen Aufbrauch der verbleibenden inspiratorischen Kapazität, zum anderen durch das als pathologisch zu wertende Erzielen einer $\dot{V} E$ unter Belastung entsprechend des theoretisch erreichbaren Atemminutenvolumens (MVV). Letzteres wird beim Lun- 


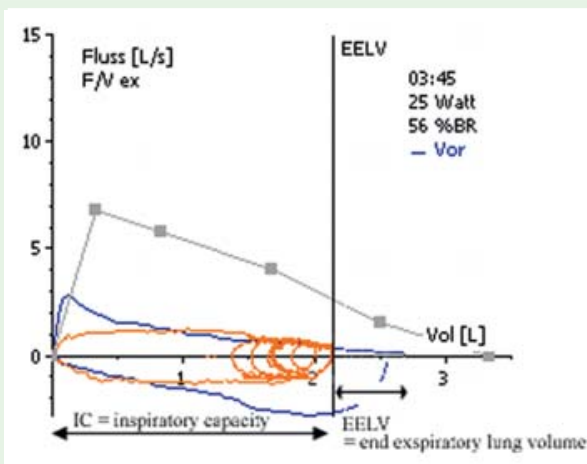

a 25 Watt

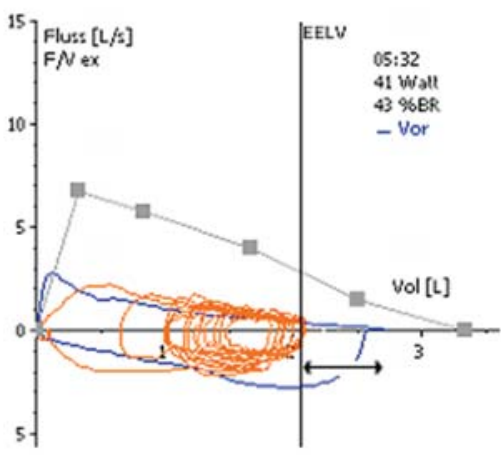

b 41 Watt

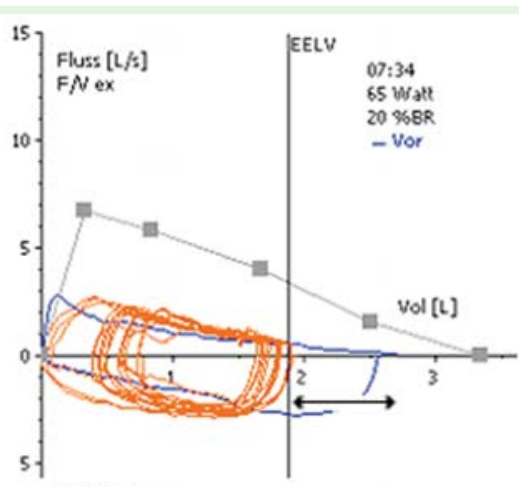

c 65 Watt

Abb.2 a-c Fluss-Volumen-Kurven einer 57-jährigen Patientin mit Asthma infolge einer Latex-Allergie (BK Nr. 4301) unter zunehmender Belastung mit Vergrößerung des EELV (von 0,57 über 0,65 zu 0,83 L) zur Inspiration hin, entsprechend einer dynamischen Lungenblähung.

Tab. 3 Einschätzung der MdE anhand der Belastungsuntersuchungen in der Begutachtung obstruktiver Atemwegserkrankungen.

\begin{tabular}{|c|c|}
\hline MdE & Belastung mit Blutgasbestimmung/Spiroergometrie \\
\hline 10 & \multirow{3}{*}{$\begin{array}{l}\text { Ergometrie: Normaler Sauerstoffpartialdruck bei hoher } \\
\text { Belastung }{ }^{1} \\
\text { Spiroergometrie: Insuffizienzkriterien }{ }^{2} \text { (siehe Erläuterungen) } \\
\text { bei hoher Belastung (bei einer } \dot{\mathrm{VO}}_{2} \text { von } 80-65 \% \text { des } \dot{\mathrm{VO}}_{2} \text {-Soll) }\end{array}$} \\
\hline 20 & \\
\hline 30 & \\
\hline 40 & \multirow{3}{*}{$\begin{array}{l}\text { Ergometrie: Verminderter Sauerstoffpartialdruck }{ }^{3} \text { bei hoher } \\
\text { oder mittlerer Belastung } \\
\text { Spiroergometrie: Insuffizienzkriterien }{ }^{2} \text { bei mittlerer } \\
\text { Belastung (bei einer } \dot{\mathrm{VO}}_{2} \text { von }<65-50 \% \text { des } \dot{\mathrm{VO}}_{2} \text {-Soll) }\end{array}$} \\
\hline 50 & \\
\hline 60 & \\
\hline 70 & \multirow{2}{*}{$\begin{array}{l}\text { Ergometrie: Verminderter Sauerstoffpartialdruck }{ }^{3} \text { bei } \\
\text { leichter Belastung }{ }^{4} \\
\text { Spiroergometrie: Insuffizienzkriterien }{ }^{3} \text { bei leichter Belastung } \\
\text { (bei einer } \dot{\mathrm{VO}}_{2} \text { von }<50 \% \text { des } \dot{\mathrm{VO}}_{2} \text {-Soll) }\end{array}$} \\
\hline 80 & \\
\hline 90 & \multirow{2}{*}{$\begin{array}{l}\text { Belastungsuntersuchung wegen Schwere der Erkrankung } \\
\text { nicht möglich }\end{array}$} \\
\hline 100 & \\
\hline
\end{tabular}

${ }^{1} 80 \%$ des Sollwertes nach Reiterer 1975 [19] werden erreicht

${ }^{2}$ Abweichung von Normwerten, v. a. von $\dot{\mathrm{VO}}_{2 \max }, \dot{\mathrm{V}} \mathrm{O}_{2, \mathrm{AT}}, \mathrm{P}_{(\mathrm{A}-\mathrm{a}), \mathrm{O} 2}, \dot{\mathrm{V} E}$ und ventilatorische Reserve, Fluss-Volumen-Kurve, Atemäquivalente

${ }^{3}$ vorrangig bei COPD/Emphysem zu erwarten; in Grenzfällen ist der standardisierte $\mathrm{P}_{\mathrm{a}, \mathrm{O} 2}$ zu verwenden [18]

${ }^{4}$ leichte Belastung: < $40 \%$ des Sollwertes nach Reiterer 1975 werden erreicht

gengesunden über die $\mathrm{FEV}_{1}$ der Ruhemessung $\times 35$ abgeschätzt. Insbesondere beim Emphysematiker mit bronchialer Instabilität wird das $\mathrm{FEV}_{1}$ im forcierten Atemmanöver niedrig gemessen; unter der eher gleichmäßigen Atmung bei Belastung können eventuell größere Ventilationsvolumina erreicht werden. Die Abschätzung des MVV wird daher in diesen Fällen mit $\mathrm{FEV}_{1} \times 40$ vorgeschlagen [11].

Auch bei nur leichtgradigem Asthma oder hyperreaktivem Bronchialsystem kann die Spiroergometrie über den Nachweis einer Strömungslimitierung ein frühes Erkrankungsstadium aufzeigen [17].

Lungenerkrankungen, die mit einer restriktiven Ventilationsstörung einhergehen, zeigen in der Spiroergometrie eine Begrenzung des Atemzugvolumens mit kompensatorischer Erhöhung der Atemfrequenz, letztendlich aber ein vermindertes Ventilationsvolumen (z.B. BK 4201 exogen-allergische Alveolitis). Bei den unter Belastung aufgezeichneten Intrabreath-Kurven kann sich die Restriktion in der aufgebrauchten inspiratorischen Kapazität zeigen, jedoch ohne begleitende dynamische Lungenblähung. Vornehmlich die restriktive Ventilationsstörung ist mit einer Gasaustauschstörung vergesellschaftet. Sie zeigt sich, wie be- reits ausgeführt, in der Erniedrigung des Sauerstoffpartialdrucks im Blut und/oder Erhöhung der alveolo-arteriellen Sauerstoffdifferenz unter Belastung. Bei beginnenden Fibrosierungen des Lungenparenchyms mit noch normaler Vitalkapazität kann letzteres auch der einzige wesentliche Befund sein (BK 4103 oder 4115).

\section{Aktuelle Empfehlungen zur MdE-Bemessung}

Zur Begutachtung asbestbedingter Lungenerkrankungen, der Silikose und arbeitsbedingter obstruktiver Atemwegserkrankungen stehen die bereits genannten Leitlinien und Begutachtungsempfehlungen [2-6] zur Verfügung. Alle enthalten eine Tabelle zur Bewertung des medizinischen Anteiles der MdE mit Angaben zu Anamnese, klinischer Symptomatik, Lungenfunktion in Ruhe, notwendiger Therapie sowie Belastungsuntersuchungen mit Blutgasen und spiroergometrischen Kriterien. Die Bewertung der Belastungsuntersuchung ist in den genannten Leitlinien und Empfehlungen weitgehend angeglichen; sie wurde zuletzt für die aktuelle Reichenhaller Empfehlung nochmals leicht modifiziert (๑ Tab.3).

Die in Tab. 3 zu berücksichtigenden Insuffizienzkriterien in der Spiroergometrie werden anhand folgender Parameter bestimmt:

- Maximale Sauerstoffaufnahme $\left(\dot{\mathrm{VO}}_{2 \max }\right)$ unterhalb $80 \%$ des Sollwertes belegt eine Leistungseinschränkung ohne Hinweis auf die Ursache.

- Sauerstoffaufnahme an der aerob-anaeroben Schwelle $\left(\dot{\mathrm{VO}}_{2, \mathrm{AT}}\right)$ unterhalb $40 \%$ des $\dot{\mathrm{V}} \mathrm{O}_{2}$-Solls zeigt eine deutliche Minderung der Ausdauerleistung.

- Erhöhte Atemäquivalente für $\mathrm{CO}_{2}\left(\dot{\mathrm{V} C O} \mathrm{C}_{2} / \dot{\mathrm{VE}}\right)$ und für $\mathrm{O}_{2}\left(\dot{\mathrm{VO}}_{2} / \dot{\mathrm{VE}}\right)$ weisen auf eine unökonomische Atmung hin; auch hier ohne Hinweis auf die Ursache.

- Erhöhte Steigung („Slope“) von V் im Verhältnis zu $\dot{\mathrm{VCO}}{ }_{2}$ : Ursache Hyperventilation (unter Ausschluss kardio-vaskulärer Erkrankungen).

- Alveolo-arterielle Sauerstoffdifferenz $\left(\mathrm{AaDO}_{2}\right)$ über $35 \mathrm{mmHg}$ : Es besteht eine Gasaustauschstörung im alveolären Bereich für Sauerstoff.

- Fehlende ventilatorische Reserve $\left(\dot{\mathrm{VE}}_{\max }-35 \times \mathrm{FEV}_{1}\right)$ am Ende der Belastung: Nachweis einer pulmonalen Ausbelastung.

- Limitierter Exspirationsfluss unter zunehmender Belastung. In der Bewertung der MdE werden die Normwerte nach Wasserman et al. [20] bzw. Hansen, Sue, Wasserman [13] entsprechend den Empfehlungen der ATS/ACCP [21] zugrunde gelegt.

Anhand eines Beispiels (siehe Kasten) wird die MdE-Bewertung erläutert. 


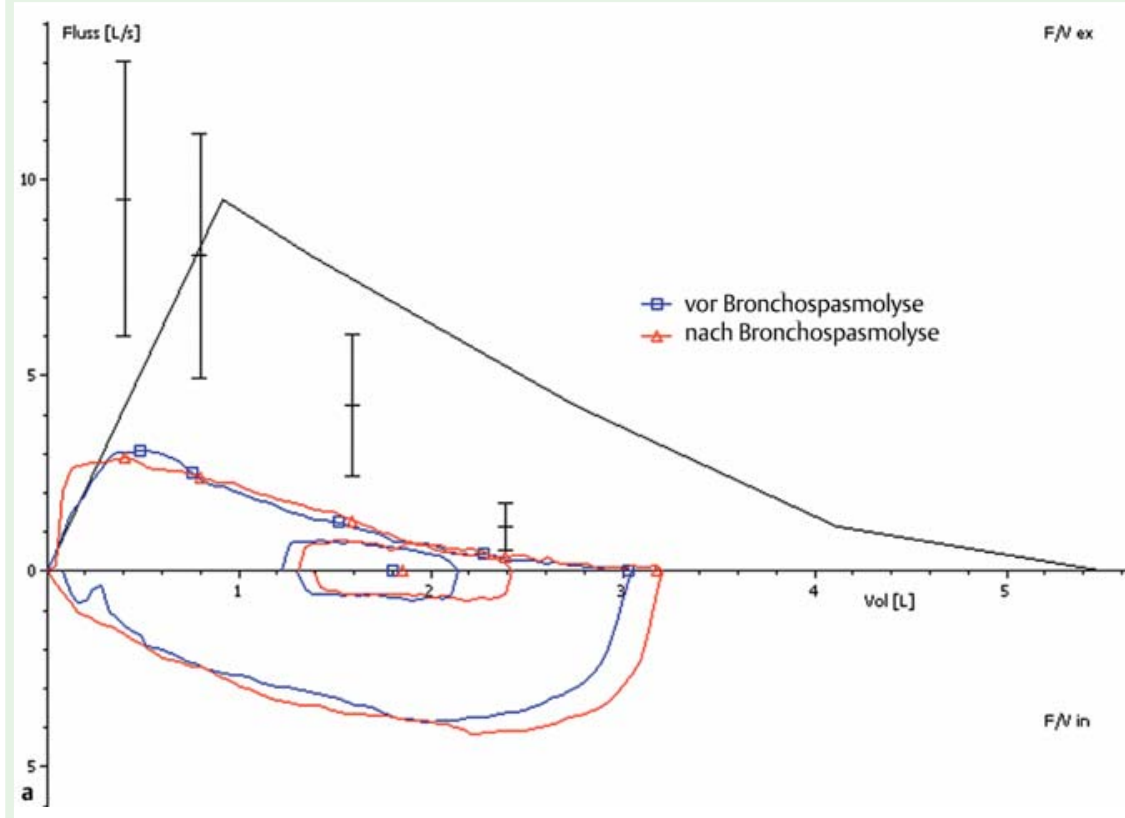

Abb. 3 a u. b Spirometrie und Bodyplethysmografie in Ruhe, vor und nach Reversibilitätstest (Werte in Klammern): VC: 3,02 L, 55\% d.S. (3,18L); FEV $_{1}: 1,80$ L, $44 \%$ d.S. $(1,85 \mathrm{~L})$; sRt: $1,16 \mathrm{kPa}^{*} \mathrm{~s}$ (1,14 kPa*s); TGV: 2,7L, 62\% d.S. (2,4L), $D_{L, C O}: 55 \%$ d. S., D $D_{L, C O} / V A: 102 \%$ d. S.

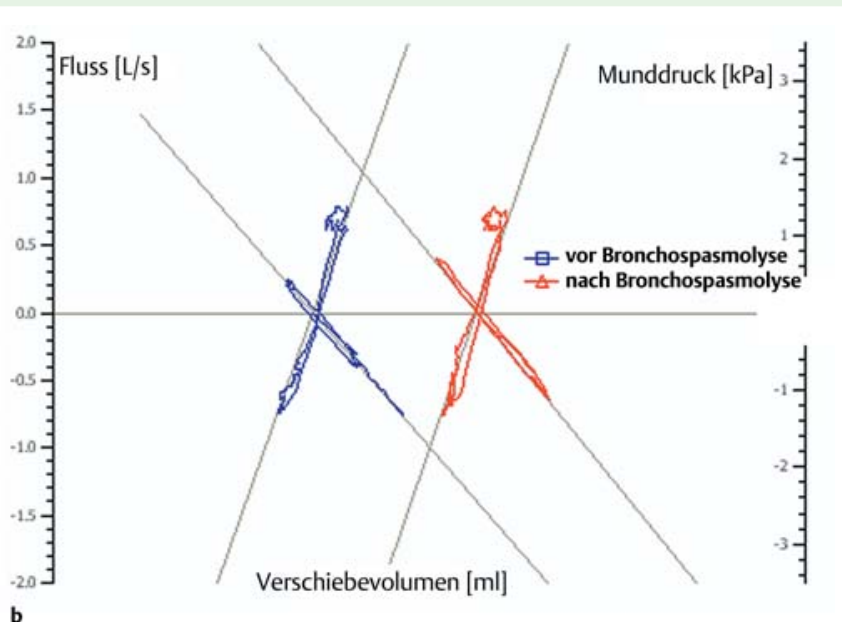

b

Lungenfunktionseinschränkungen mit einer eingeschränkten maximalen Sauerstoffaufnahme und verminderter Leistungsfähigkeit unter Ausschöpfung der pulmonalen Reserven führen in der Regel zu einer MdE von $\geq 20 \%$.

\section{Schlussfolgerungen und Ausblick}

Die Spiroergometrie hat als differenzialdiagnostisches Instrument in der Arbeitsmedizin in den letzten Jahren Anerkennung gewonnen und wird in der Begutachtung von asbestbedingten Erkrankungen, der Silikose und arbeitsbedingten obstruktiven Atemwegserkrankungen [4-6] zunehmend eingesetzt. Für die Diagnosesicherung einer pulmonalen Erkrankung im sog. „Vollbeweis“, insbesondere wenn diese nur durch Dyspnoe bei Belastung manifest wird, ist die Sicherung funktioneller Einschränkungen auch bereits im Frühstadium der Erkrankung notwendig. Die Spiroergometrie ist weiterhin bedeutsam zur Einschätzung der MdE und für die Differenzierung zwischen einerseits berufsbedingten pulmonalen Funktionseinschränkungen und andererseits berufskrankheitsunabhängigen Leistungsminderungen durch Herz/Kreislauf- und neuromuskuläre Erkrankungen.
Beispiel

Arbeitsmedizinische Begutachtung eines 56-jährigen Patienten mit einer Silikose (Berufskrankheit Nr.4101) Gießereiarbeiter am Hochofen von 1970-1989, er habe Quarzsand in Formen eingefüllt mit starker Staubentwicklung, Nieraucher. Die BK 4101 wurde im Jahr 2000 vom Unfallversicherungsträger anerkannt, die MdE ist zu bemessen. Zum Begutachtungszeitpunkt 11/2010 Luftnot bei geringen körperlichen Belastungen, wie z.B. Körperpflege, langsames Gehen in der Ebene möglich. Morgens Husten mit gelblichem Auswurf; Medikation mit langwirksamen $\beta$-Sympathomimetika, Corticoid und Tiotropium inhalativ; diese wurden am Morgen des Untersuchungstages ausgesetzt; $2 \mathrm{~L} \mathrm{O}_{2} / \mathrm{min}$ bei starker Belastung.

MdE-Bewertung. In der Bewertung des medizinischen Anteiles der MdE wurden die Beschwerden, die mittelschwere kaum reversible Atemwegsobstruktion (dargestellt in $\bullet$ Abb. 3), die Medikation und die Spiroergometrie ( Abb.4) mit eingeschränkter körperlicher Belastbarkeit aufgrund der in der Fluss-Volumen-Kurve deutlich erkennbaren Strömungslimitierung mit dynamischer Lungenblähung ( $\bullet$ Abb.5) und der unter Belastung erhöhten $\mathrm{AaDO}_{2}$ berücksichtigt. Letztgenannte Insuffizienzzeichen zeigten sich ab ca. 60 Watt, entsprechend $1,45 \mathrm{~L} \mathrm{VO}_{2}$ oder $56 \%$ des $\dot{\mathrm{VO}}_{2 \mathrm{max}}$-Soll - die Bewertung der Spiroergometrie nach $\bullet$ Tab. 2 entspricht somit einer MdE im Bereich 40-60\%. Unter Berücksichtigung aller genannten Kriterien wurde integrativ für die gemischt obstruktiv-restriktive Ventilationsstörung und Gasaustauschstörung die Festsetzung einer MdE von 50\% empfohlen.

In der Beurteilung von Lungenerkrankungen mit restriktiver Ventilationsstörung ist die Bewertung einer Gasaustauschstörung unter Belastung mit Berücksichtigung der Ventilationsparameter (v.a. $\mathrm{AaDO}_{2}$ ) bedeutsam. Die alleinige Bestimmung der Blutgase unter Belastung würde in ausgeprägten Fällen auch die Gasaustauschstörung darstellen, jedoch wäre die kompensierende Ventilation nicht ausreichend berücksichtigt. Die Begutach- 

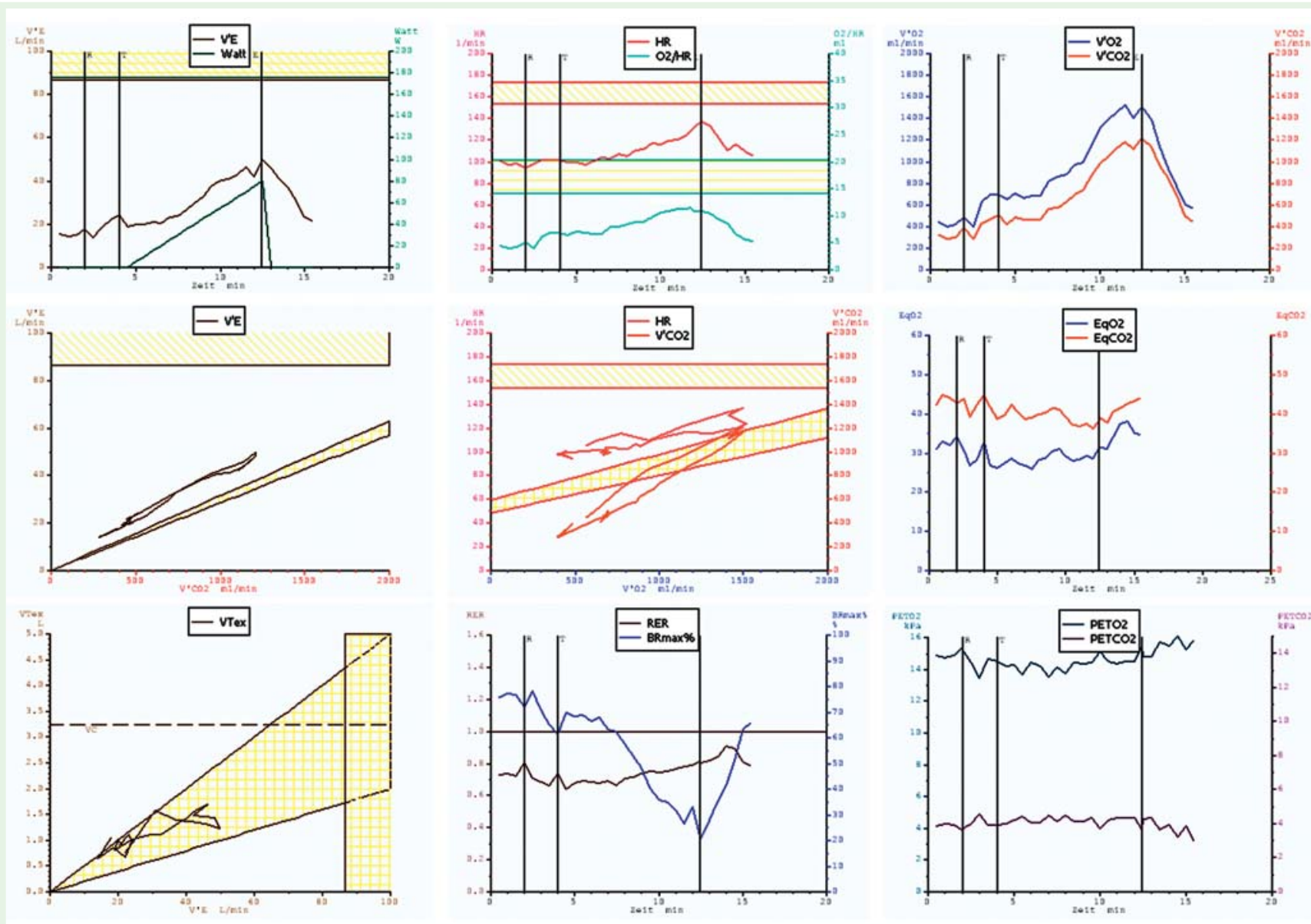

Abb.4 Neun-Felder-Grafik nach Wasserman [20]; die Insuffizienzkriterien entsprechend der $\bullet$ Tab. 3 werden folgend erläutert: $\dot{V}_{2 m a x}: 65 \%$ des Solls, die aerob-anaerobe Schwelle wurde nicht erreicht. Sauerstoffpartialdruck in Ruhe $78 \mathrm{mmHg}$ (unterer Grenzwert: 71,5 mmHg), $\mathrm{P}_{\mathrm{a}, \text { co2: }}: 37 \mathrm{mmHg}$, unter Belastung $\mathrm{P}_{\mathrm{a}, 02}: 70 \mathrm{mmHg}, \mathrm{P}_{\mathrm{a}, \mathrm{CO} 2}: 35 \mathrm{mmHg} ; \mathrm{AaDO}_{2}$ in Ruhe $21,8 \mathrm{mmHg}$, unter Belastung (bei $56 \mathrm{~W}$ ) $35,3 \mathrm{mmHg}$ (normal bis $35 \mathrm{mmHg}$ ). Herzfrequenz maximal 133 Schläge/min, keine kardiale Ausbelastung. Abbruch wegen Strömungslimitierung ( $\boldsymbol{A}$ Abb. 5), geringe pulmonale Reserve bei Belastungsende, dies bedeutet die Differenz zwischen $\dot{\mathrm{V}} \mathrm{E}_{\max }$ von $48 \mathrm{~L} /$ min und theoretisch erreichbarem $\dot{V} E$ von $63 \mathrm{~L} /$ min (berechnet aus FEV Von $_{1}, 8 \mathrm{~L}^{*} 35$ ). EqCO $\mathrm{Eq}_{2} 36$ leicht erhöht (normal bis 32) als Hinweis auf unökonomische Atmung und Hyperventilation.

tungsempfehlungen der Unfallversicherungsträger [4,5] empfehlen hier eine Berechnung des $\mathrm{P}_{\mathrm{a}, 02}$ unter Zuhilfenahme einer „Standardisierung“ nach Dieckmann und Smidt [18], die unter Einbeziehung des $\mathrm{P}_{\mathrm{a}, \mathrm{CO} 2}$ auch die Ventilation und insbesondere die Hyperventilation berücksichtigt. Diese Formel wurde jedoch nicht für Belastungsparameter entwickelt und hat daher diesbezüglich eine eingeschränkte Aussagekraft. Im Falle restriktiver Ventilationsstörungen zeigte sich in eigenen Untersuchungen aber eine gute Korrelation der $\mathrm{AaDO}_{2}$ und des „standardisierten“ $\mathrm{P}_{\mathrm{a}, 02}$ [22]. Eine abschließende Bewertung ist zurzeit noch nicht möglich.

In der Begutachtungsempfehlung zu obstruktiven Atemwegserkrankungen wird die Spiroergometrie im Unterschied zum vorherigen Merkblatt [6] als optionale Untersuchung in der Differenzialdiagnostik der Belastungsdyspnoe aufgeführt. In der Beurteilung von Belastbarkeit und Einschränkungen durch eine Obstruktion kann die spiroergometrische Untersuchung genauer graduieren und so die eigentliche Ursache der verminderten Belastbarkeit eingrenzen, z.B. Strömungslimitierung oder Trainingsmangel. Unverzichtbar ist hier die Aufzeichnung der Fluss-VolumenKurve unter Belastung in Erweiterung der üblichen 9-Felder-Grafik nach Wasserman [20]. Lo Russo et al. [23] zeigten bereits 1993 eine niedrige Korrelation von Leistungsfähigkeit $\left(\dot{\mathrm{VO}}_{2 \max }\right)$ und Graduierung der Obstruktion in Ruhe $\left(\mathrm{FEV}_{1}\right)$. Auch in der Begut- achtung obstruktiver Atemwegserkrankungen sollte daher zur Einschätzung der MdE auf die Spiroergometrie nicht verzichtet werden. Darüber hinaus eignet sich die Spiroergometrie zur Frühdetektion einer eingeschränkten Belastbarkeit aufgrund eines hyperreaktiven Bronchialsystems als Ergänzung zum unspezifischen bronchialen Provokationstest [17]. Sie stellt somit einen wesentlichen Baustein in der arbeitsmedizinischen Begutachtung von Berufskrankheiten mit Objektivierung der körperlichen Belastbarkeit und Einordnung der Ursache der Leistungsminderung dar.

In der Eignungsbeurteilung für berufliche Tätigkeiten mit hoher körperlicher Belastung und Tragen von schwerem Atemschutz wird bisher vornehmlich die Ergometrie mit relativ starr vorgegebenen Pulsfrequenz- zu Watt- und Körpergewichts-Relationen verwendet. Die Spiroergometrie ermöglicht es, hier zusätzliche Informationen zur Dauerleistungsfähigkeit und zur Ursache möglicher Belastungseinschränkungen zu erhalten. Aus diesen Gründen und da der Aufwand einer Spiroergometrie in der Durchführung im Vergleich zu einer Ergometrie - nach Einarbeitung - nicht wesentlich höher ist, halten wir die Spiroergometrie in der Eignungsuntersuchung von Personen mit hoher körperlicher Beanspruchung, Tragen von Druckluft-Atemgeräten oder Arbeiten in Druckluft und gleichzeitig hoher Gefährdung (Berufsfeuerwehr, Berufstaucher und Druckluftarbeiter) für notwendig. 


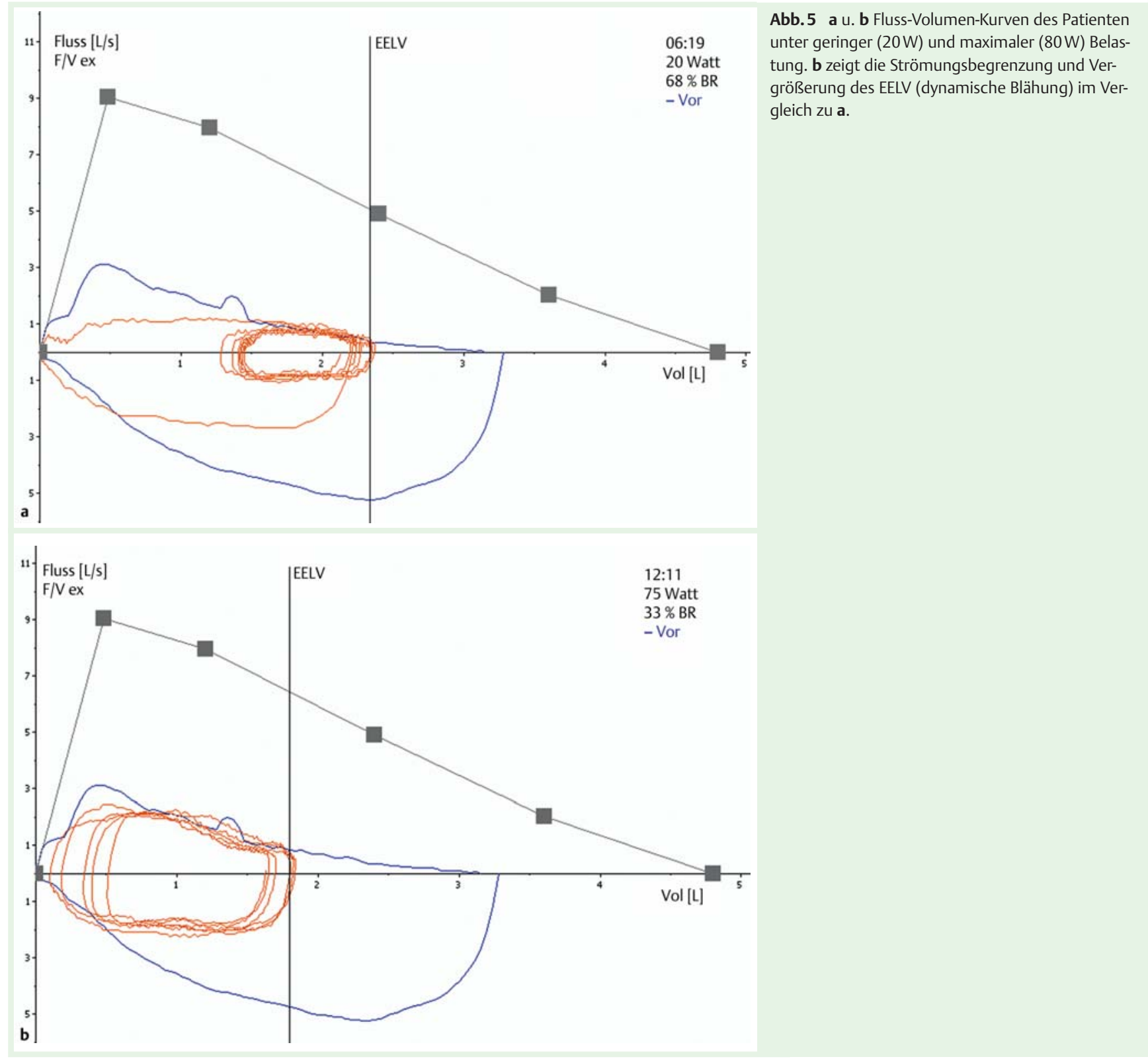

\section{Interessenkonflikt}

$\nabla$

Die Autoren geben an, dass kein Interessenkonflikt besteht.

\section{Literatur}

1 Ochmann U, Preisser A, Nowak D. Pneumologische Belastungsuntersuchungen in der Arbeitsmedizin. Der Pneumologe 2011; 8: 92 -97

2 Deutsche Gesellschaft für Pneumologie und Beatmungsmedizin, Deutsche Gesellschaft für Arbeitsmedizin und Umweltmedizin (DGAUM). Quarzstaublungenerkrankung (Silikose). Diagnostik und Begutachtung der Berufskrankheit Nr. 4101 (Reg. Nr.: 020-010, Entwicklungsstufe: S2). http://www.awmf.org/leitlinien/detail/1l/020-010.html (02.08.2011)

3 Deutsche Gesellschaft für Pneumologie und Beatmungsmedizin, Deutsche Gesellschaft für Arbeitsmedizin und Umweltmedizin (DGAUM). Diagnostik und Begutachtung asbestbedingter Berufskrankheiten. (Reg. Nr.: 002-038, Entwicklungsstufe: S2k). http://www.awmf.org/ leitlinien/detail/11/002-038.html (02.08.2011)

4 Deutsche Gesetzliche Unfallversicherung (DGUV). Empfehlung für die Begutachtung asbestbedingter Berufskrankheiten. Falkensteiner Empfehlung. Februar 2011. http://publikationen.dguv.de/dguv/pdf/10002/ falkensteinerempfehlung.pdf (02.08.2011)
5 Deutsche Gesetzliche Unfallversicherung (DGUV). Empfehlung für die Begutachtung von Quarzstaublungenerkrankungen (Silikosen). Bochumer Empfehlung. Juni 2011. http://publikationen.dguv.de/dguv/ pdf/10002/bochumer.pdf (02.08.2011)

6 Hauptverband der gewerblichen Berufsgenossenschaften (HVBG). Reichenhaller Merkblatt: Begutachtungsempfehlungen für die Berufskrankheiten der Nrn. 1315 (ohne Alveolitis), 4301 und 4302 der Anlage zur BKV. April 2006. http://www.dguv.de/inhalt/medien/bestellung/documents/reichenhall.pdf

7 Preisser A, Baur X. Vorschläge zur Aktualisierung des „Reichenhaller Merkblatt - Begutachtungsempfehlungen für die Berufskrankheiten der Nrn. 1315 (ohne Alveolitis), 4301 und 4302 der Anlage zur BKV“. Pneumologie 2008; 62: 491 - 493

8 Bundesanstalt für Arbeitsschutz und Arbeitsmedizin (BAuA). Liste der Berufskrankheiten. Anlage 1 zur Berufskrankheiten-Verordnung (BKV) in der Fassung der Zweiten Verordnung zur Änderung der Berufskrankheiten-Verordnung vom 11. Juni 2009. http://www.baua.de/de/ Publikationen/Faltblaetter/F3.pdf (02.08.2011)

9 Reiterer W, Bachl N. Kriterien der körperlichen Leistungsfähigkeit. Wien Med Wochenschr Suppl 1977; 42: 1-19

10 Wasserman K, Sue DY. Coupling of external to cellular respiration. In: Wasserman K. Exercise gas exchange heart disease. Armonk, NY: Futura Publishing; 1996 
11 Kroidl RF, Schwarz S, Lehnigk B. Kursbuch Spiroergometrie: Technik und Befundung verständlich gemacht. Stuttgart: Thieme; 2009

12 Kroidl RF. Spiroergometrie und Begutachtung. Atemwegs- und Lungenkrankheiten 2009; 35: 18-32

13 Hansen JE, Sue DY, Wasserman K. Predicted Values for Clinical Exercise Testing: Including Pathophysiology and Clinical Applications. Am Rev Respir Dis 1984; 129: 49-55

14 Koch B, Schäper C, Ittermann $T$ et al. Reference values for cardiopulmonary exercise testing in healthy volunteers: The SHIP study. Eur Respir J 2009; 33: 389-397

15 Deutsche Gesetzliche Unfallversicherung (DGUV). Berufsgenossenschaftliche Grundsätze für arbeitsmedizinische Vorsorgeuntersuchungen. 5. Aufl. Stuttgart: Gentner; 2010

16 Heblich F. Belastung von Atemschutzgeräteträgern - sind gestufte Anforderungen an die Leistungsfähigkeit gerechtfertigt? Fachsanitätszentrum Kiel der Bundesmarine, Arztgruppe Betriebsmedizin; Persönliche Mitteilung
17 Martinez FJ, Stanopoulos I, Acero R et al. Graded comprehensive cardiopulmonary exercise testing in the evaluation of dyspnea unexplained by routine evaluation. Chest 1994; 105: $168-174$

18 Diekmann $M$, Smidt $U$. Berechnung eines Standard- $\mathrm{PaO}_{2}$ in Analogie zum Standard-Bikarbonat. Atemwegs- und. Lungenerkrankungen 1984; 10: $248-250$

19 Reiterer W. Methodik eines rektangulär-triangulären Belastungstestes. Herz/Kreislauf 1975; 7: 457-462

20 Wasserman K, Hansen JE, Sue DY et al. Principles of Exercise Testing and Interpretation. Baltimore: Lippincott Raven; 2004

21 American Thoracic Society (ATS) and the American College of Chest Physicians (ACCP). Statement on Cardiopulmonary Exercise Testing. Am J Respir Crit Care Med 2003; 167: 211 - 277

22 Preisser A, Manuwald U, Barbinova $L$ et al. Blutgase unter Belastung im Vergleich mit der alveolo-arteriellen Sauerstoffdifferenz. Pneumologie 2010; 64: 170

23 LoRusso TJ, Belman MJ, Elashoff JD et al. Prediction of maximal exercise capacity in obstructive and restrictive pulmonary disease. Chest 1993; 104: $1748-1754$ 